\title{
The Goldman-Rota identity and the Grassmann scheme
}

\author{
Murali K. Srinivasan \\ Department of Mathematics \\ Indian Institute of Technology, Bombay \\ Powai, Mumbai 400076, India \\ mks@math.iitb.ac.in, murali.k.srinivasan@gmail.com
}

Submitted: Jul 8, 2013; Accepted: Feb 15, 2014; Published: Feb 21, 2014

Mathematics Subject Classifications: 05E25, 05E30.

To the memory of Kaala, BMW, and $\infty$

\begin{abstract}
We inductively construct an explicit (common) orthogonal eigenbasis for the elements of the Bose-Mesner algebra of the Grassmann scheme.

The key step is a constructive, linear algebraic interpretation of the GoldmanRota recurrence for the number of subspaces of a finite vector space. This interpretation shows that the up operator on subspaces has an explicitly given recursive structure.

Using the interpretation above we inductively construct an explicit orthogonal symmetric Jordan basis with respect to the up operator and write down the singular values, i.e., the ratio of the lengths of the successive vectors in the Jordan chains. The collection of all vectors in this basis of a fixed rank $m$ forms a (common) orthogonal eigenbasis for the elements of the Bose-Mesner algebra of the Grassmann scheme of $m$-dimensional subspaces. We also pose a bijective proof problem on the spanning trees of the Grassmann graphs.
\end{abstract}

\section{Introduction}

This paper presents constructive and explicit proofs of two basic linear algebraic results on the subspace lattice. We begin by recalling two important existence results on subspaces.

Let $P$ be a finite graded poset with rank function $r: P \rightarrow \mathbb{N}=\{0,1,2, \ldots\}$ (our poset terminology follows [29]). The rank of $P$ is $r(P)=\max \{r(x): x \in P\}$ and, for 
$i=0,1, \ldots, r(P), P_{i}$ denotes the set of elements of $P$ of rank $i$. For a subset $S \subseteq P$, we set $\operatorname{rankset}(S)=\{r(x): x \in S\}$. A symmetric chain in a graded poset $P$ is a sequence

$$
\left(p_{1}, p_{2}, \ldots, p_{h}\right)
$$

of elements of $P$ such that $p_{i}$ covers $p_{i-1}$, for $i=2, \ldots, h$ and $r\left(p_{1}\right)+r\left(p_{h}\right)=r(P)$, if $h \geqslant 2$, or else $2 r\left(p_{1}\right)=r(P)$, if $h=1$. A symmetric chain decomposition (SCD) of a graded poset $P$ is a decomposition of $P$ into pairwise disjoint symmetric chains.

Let $B(n)$ denote the collection of all subsets of the set $\{1,2, \ldots, n\}$. Partially order $B(n)$ by inclusion. Let $\mathbb{F}_{q}^{n}$ denote the $n$-dimensional vector space of all column vectors of length $n$ over $\mathbb{F}_{q}$ and let $B_{q}(n)$ denote the collection of all subspaces of $\mathbb{F}_{q}^{n}$. Partially order $B_{q}(n)$ by inclusion. Define $[k]_{q}=1+q+q^{2}+\cdots+q^{k-1}, k \in \mathbb{N}$. For $0 \leqslant k \leqslant n$, the q-binomial coefficient

$$
\left[\begin{array}{c}
n \\
k
\end{array}\right]_{q}=\frac{[1]_{q}[2]_{q} \cdots[n]_{q}}{[1]_{q} \cdots[k]_{q}[1]_{q} \cdots[n-k]_{q}}
$$

denotes the number of $k$-dimensional subspaces in $B_{q}(n)$ and the total number of subspaces is the Galois number

$$
G_{q}(n)=\sum_{k=0}^{n}\left[\begin{array}{l}
n \\
k
\end{array}\right]_{q}
$$

The following result was proved in [13] using network flow techniques.

Theorem 1.1. There exists a symmetric chain decomposition of the graded poset $B_{q}(n)$.

Although Theorem 1.1 is interesting in itself one of its main motivations was that the existence of a SCD of a graded poset $P$ is a stronger property than $P$ being strongly Sperner, a fact that was already known for $B_{q}(n)$ (see [9]).

Theorem 1.1 has a linear analog, which we now state.

For a finite set $S$, let $V(S)$ denote the complex vector space with $S$ as basis. Let $v=\sum_{x \in S} \alpha_{x} x, \alpha_{x} \in \mathbb{C}$ be an element of $V(S)$. By the support of $v$ we mean the subset $\left\{x \in S: \alpha_{x} \neq 0\right\}$.

Let $P$ be a graded poset with $n=r(P)$. Then we have

$$
V(P)=V\left(P_{0}\right) \oplus V\left(P_{1}\right) \oplus \cdots \oplus V\left(P_{n}\right)
$$

(vector space direct sum). An element $v \in V(P)$ is homogeneous if $v \in V\left(P_{i}\right)$ for some $i$, and if $v \neq 0$, we extend the notion of rank to nonzero homogeneous elements by writing $r(v)=i$. Given an element $v \in V(P)$, write $v=v_{0}+\cdots+v_{n}$, where $v_{i} \in V\left(P_{i}\right), 0 \leqslant i \leqslant n$. We refer to the $v_{i}$ as the homogeneous components of $v$. A subspace $W \subseteq V(P)$ is homogeneous if it contains the homogeneous components of each of its elements. For a homogeneous subspace $W \subseteq V(P)$ we set

$$
\operatorname{rankset}(W)=\{r(v): v \text { is a nonzero homogeneous element of } W\} \text {. }
$$


The up operator $U: V(P) \rightarrow V(P)$ is defined, for $x \in P$, by $U(x)=\sum_{y} y$, where the sum is over all $y$ covering $x$. We denote the up operator on $V\left(B_{q}(n)\right)$ by $U_{n}$. For a finite vector space $X$ over $\mathbb{F}_{q}$ we denote by $B_{q}(X)$ the set of all subspaces of $X$ and we denote by $U_{X}$ the up operator on $V\left(B_{q}(X)\right)$.

Let $\langle$,$\rangle denote the standard inner product on V(P)$, i.e., $\langle x, y\rangle=\delta(x, y)$ (Kronecker delta), for $x, y \in P$. The length $\sqrt{\langle v, v\rangle}$ of $v \in V(P)$ is denoted $\|v\|$. We give $V\left(B_{q}(n)\right)$ (and $V\left(B_{q}(X)\right)$ for a finite vector space $X$ over $\mathbb{F}_{q}$ ) the standard inner product.

Let $P$ be a finite graded poset with rank function $r$. A graded Jordan chain in $V(P)$ is a sequence

$$
s=\left(v_{1}, \ldots, v_{h}\right)
$$

of nonzero homogeneous elements of $V(P)$ such that $U\left(v_{i-1}\right)=v_{i}$, for $i=2, \ldots h$, and $U\left(v_{h}\right)=0$ (note that the elements of this sequence are linearly independent, being nonzero and of different ranks). We say that $s$ starts at rank $r\left(v_{1}\right)$ and ends at rank $r\left(v_{h}\right)$. A graded Jordan basis of $V(P)$ is a basis of $V(P)$ consisting of a disjoint union of graded Jordan chains in $V(P)$. The graded Jordan chain (1) is said to be a symmetric Jordan chain (SJC) if the sum of the starting and ending ranks of $s$ equals $r(P)$, i.e., $r\left(v_{1}\right)+r\left(v_{h}\right)=r(P)$ if $h \geqslant 2$, or $2 r\left(v_{1}\right)=r(P)$ if $h=1$. A symmetric Jordan basis (SJB) of $V(P)$ is a basis of $V(P)$ consisting of a disjoint union of symmetric Jordan chains in $V(P)$.

The following result was proved in [30] using [8].

Theorem 1.2. There exists an orthogonal symmetric Jordan basis $T_{q}(n)$ of $V\left(B_{q}(n)\right)$.

Let $0 \leqslant k \leqslant n / 2$ and let $\left(x_{k}, \ldots, x_{n-k}\right)$ be any symmetric Jordan chain in $T_{q}(n)$ starting at rank $k$ and ending at rank $n-k$. Then we have, for $k \leqslant u<n-k$,

$$
\frac{\left\|x_{u+1}\right\|}{\left\|x_{u}\right\|}=\sqrt{q^{k}[u+1-k]_{q}[n-k-u]_{q}} .
$$

See [26] for a proof of Theorem 1.2 based on the $\mathfrak{s l}(2, \mathbb{C})$ method [22]. Very closely related results are shown in $[7,28,20,1]$. The numbers on the right hand side of $(2)$ are called the singular values of the up operator. These are important for applications.

Theorem 1.2 has several applications. In [27] we showed that the commutant of the $G L\left(n, \mathbb{F}_{q}\right)$ action on $V\left(B_{q}(n)\right)$ block diagonalizes with respect to the orthonormal basis given by the normalization of $T_{q}(n)$ and we used (2) to make this block diagonalization explicit, thereby obtaining a $q$-analog of the formula from [23] for explicit block diagonalization of the commutant of the symmetric group action on $V(B(n))$. This includes, as a special case, a formula for the eigenvalues of the elements of the Bose-Mesner algebra of the Grassmann scheme [6, 5]. For other approaches to explicit block diagonalization see $[23,19,20,1]$, the first and last of which give applications to bounds on codes using semidefinite programming. In [26] we used (2) to give a positive combinatorial formula for the number of spanning trees of the $q$-analog of the $n$-cube and to show that the Laplacian eigenvalues of the Grassmann graphs, known in principle since [6], admit an 
elegant closed form. For another approach to the Laplacian eigenvalues of the Grassmann graphs see [20]. At the end of this paper we pose a bijective proof problem on spanning trees of the Grassmann graphs.

It is natural to ask for a constructive proof of Theorem 1.1 and this was done in the beautiful paper [31]. The underlying premise of this paper is that it is equally natural to ask for a constructive proof of Theorem 1.2. The problem of constructing an explicit SJB should be put on an equal footing with the problem of constructing an explicit SCD ("More people should get used to considering formal sums of combinatorial objects as being as natural to deal with as individual objects", quote from [2]) and in Section 3 we prove the following result.

Theorem 1.3. There is an algorithm to inductively construct an explicit orthogonal symmetric Jordan basis $J_{q}(n)$ of $V\left(B_{q}(n)\right)$. When expressed in the standard basis the vectors in $J_{q}(n)$ have coefficients that are integral multiples of qth roots of unity. In particular, the coefficients are integral when $q=2$.

Let $0 \leqslant k \leqslant n / 2$ and let $\left(x_{k}, \ldots, x_{n-k}\right)$ be any symmetric Jordan chain in $J_{q}(n)$ starting at rank $k$ and ending at rank $n-k$. Then we have, for $k \leqslant u<n-k$,

$$
\frac{\left\|x_{u+1}\right\|}{\left\|x_{u}\right\|}=\sqrt{q^{k}[u+1-k]_{q}[n-k-u]_{q}} .
$$

A standard argument (recalled in Section 3) shows that the set $\left\{v \in J_{q}(n): r(v)=m\right\}$ forms a common orthogonal eigenbasis for the elements of the Bose-Mesner algebra of the Grassmann scheme of $m$-dimensional subspaces. Explicit eigenvectors for the Johnson scheme (the $q=1$ case of the Grassmann scheme) have been well studied. See, for example, $[12,11,18,24,25]$. The approach in $[12,11,18,24]$ produces integral eigenvectors with coefficients in $\{-1,0,1\}$ while the approach in [25] produces orthogonal integral eigenvectors, with coefficients not necessarily in $\{-1,0,1\}$ (as remarked in [18] it is not possible to simultaneously satisfy both requirements, i.e., orthogonality and coefficients in $\{-1,0,1\})$. Substituting $q=1$ in Theorem 1.3 we recover the explicit orthogonal SJB of $V(B(n))$ constructed in [25]. This basis was given a representation theoretic characterization in [25], namely, that it is the canonically defined symmetric Gelfand-Tsetlin basis of $V(B(n))$. Similarly, the basis $J_{q}(n)$ should also be studied from a representation theoretic viewpoint. We hope to return to this later.

Our proof of Theorem 1.3 proceeds via another constructive result on the recursive structure of the up operator on subspaces. It is an elementary observation that the up operator (or equivalently, incidence matrices) on subsets of a $n+1$-set can be built from two copies of the up operator on subsets of a $n$-set. We extend this inductive approach to the subspace lattice. A classical identity of Goldman and Rota suggests that the up operator on subspaces of a $n+1$-dimensional vector space over $\mathbb{F}_{q}$ can be built from two copies of the up operator in dimension $n$ and $q^{n}-1$ copies of the up operator in dimension $n-1$. Let us make this precise.

We have $|B(n)|=2^{n}$ and the identity $2^{n+1}=2 \cdot 2^{n}$ has the following poset theoretic 
interpretation: we can write $B(n+1)$ as a disjoint union $B(n+1)=B(n) \cup[\{n+$ $1\},\{1, \ldots, n+1\}]$, where the interval $[\{n+1\},\{1, \ldots, n+1\}]$ is order isomorphic to $B(n)$.

The Goldman-Rota identity $[10,17,21]$ is the recursion

$$
G_{q}(n+1)=2 G_{q}(n)+\left(q^{n}-1\right) G_{q}(n-1), n \geqslant 1, \quad G_{q}(0)=1, G_{q}(1)=2 .
$$

We identify $\mathbb{F}_{q}^{n}$ with the subspace of all vectors in $\mathbb{F}_{q}^{n+1}$ with last component zero. Put $t=q^{n}-1$. Motivated by the $B(n)$ case we can ask for the following poset theoretic interpretation of (4): is it possible to write $B_{q}(n+1)$ as a disjoint union

$$
B_{q}(n+1)=B_{q}(n) \cup S_{0} \cup S_{1} \cup \cdots \cup S_{t},
$$

where $S_{0}, \ldots, S_{t}$ are intervals in $B_{q}(n+1)$, with $S_{0}$ order isomorphic to $B_{q}(n)$ and $S_{1}, \ldots, S_{t}$ order isomorphic to $B_{q}(n-1)$. At least for $q=2$ and $n \geqslant 4$ the answer is no, as shown in [14].

We show that we can get a poset theoretic interpretation of (4) by considering a linear analog of (5). Moreover, the linear analog of the decomposition (5) can be explicitly given.

Let $(V, f)$ be a pair consisting of a finite dimensional vector space $V$ (over $\mathbb{C}$ ) and a linear operator $f$ on $V$. Let $(W, g)$ be another such pair. By an isomorphism of pairs $(V, f)$ and $(W, g)$ we mean a linear isomorphism $\theta: V \rightarrow W$ such that $\theta(f(v))=g(\theta(v)), v \in V$.

We prove the following result on the recursive structure of the pair $\left(V\left(B_{q}(n)\right), U_{n}\right)$ in Section 2. This result is used in Section 3 to construct the orthogonal SJB $J_{q}(n)$.

Theorem 1.4. Set $t=q^{n}-1$. There is an explicit orthogonal direct sum decomposition

$$
V\left(B_{q}(n+1)\right)=V\left(B_{q}(n)\right) \oplus W(0) \oplus W(1) \oplus \cdots \oplus W(t)
$$

where

(i) $W(0), \ldots, W(t)$ are $U_{n+1}$-closed (i.e., closed under the action of $U_{n+1}$ ) homogeneous subspaces of $V\left(B_{q}(n+1)\right)$ with rankset $(W(0))=\{1, \ldots, n+1\}$ and $\operatorname{rankset}(W(i))=$ $\{1, \ldots, n\}$, for $i=1, \ldots, t$.

(ii) $V\left(B_{q}(n)\right) \oplus W(0)$ is $U_{n+1}$-closed and there is an explicit linear map $\theta_{n}: V\left(B_{q}(n)\right) \rightarrow$ $W(0)$ that is an isomorphism of pairs $\left(V\left(B_{q}(n)\right), q U_{n}\right)$ and $\left(W(0), U_{n+1}\right)$, sending homogeneous elements to homogeneous elements, increasing rank by one and satisfying

$$
\begin{aligned}
U_{n+1}(v) & =U_{n}(v)+\theta_{n}(v), v \in V\left(B_{q}(n)\right), \\
\left\langle\theta_{n}(w), \theta_{n}(v)\right\rangle & =q^{n-k}\langle w, v\rangle, w, v \in V\left(B_{q}(n)_{k}\right), 0 \leqslant k \leqslant n .
\end{aligned}
$$

(iii) For $i=1, \ldots, t$ there $i s$ an explicit linear map $\gamma_{n-1}(i): V\left(B_{q}(n-1)\right) \rightarrow W(i)$ that is an isomorphism of pairs $\left(V\left(B_{q}(n-1)\right), U_{n-1}\right)$ and $\left(W(i), U_{n+1}\right)$, sending homogeneous elements to homogeneous elements, increasing rank by one and satisfying

$$
\left\langle\gamma_{n-1}(i)(w), \gamma_{n-1}(i)(v)\right\rangle=q^{n+k}\langle w, v\rangle, w, v \in V\left(B_{q}(n-1)_{k}\right), 0 \leqslant k \leqslant n-1 .
$$


Remark For $v \in V\left(B_{q}(n)_{k}\right), 0 \leqslant k \leqslant n, w \in V\left(B_{q}(n-1)_{k}\right), 0 \leqslant k \leqslant n-1, i=1, \ldots t$ set

$$
\begin{aligned}
\theta_{n}^{\prime}(v) & =\frac{\theta_{n}(v)}{q^{(n-k) / 2}}, \\
\gamma_{n-1}(i)^{\prime}(v) & =\frac{\gamma_{n-1}(i)(v)}{q^{(n+k) / 2}} .
\end{aligned}
$$

We may check that $\theta_{n}^{\prime}$ is an isomorphism of pairs $\left(V\left(B_{q}(n)\right), \sqrt{q} U_{n}\right)$ and $\left(W(0), U_{n+1}\right)$ that preserves inner products and satisfies

$$
U_{n+1}(v)=U_{n}(v)+q^{(n-k) / 2} \theta_{n}^{\prime}(v), v \in V\left(B_{q}(n)_{k}\right), 0 \leqslant k \leqslant n .
$$

Similarly, it may be checked that $\gamma_{n-1}(i)^{\prime}$ is an inner product preserving isomorphism of pairs $\left(V\left(B_{q}(n-1)\right), \sqrt{q} U_{n-1}\right)$ and $\left(W(i), U_{n+1}\right)$. However, certain computations (notably in the proofs of Theorems 1.3 and 2.5 below) then become slightly cumbersome due to the presence of many square roots. Therefore we prefer the formulation above where the maps $\theta_{n}$ and $\gamma_{n-1}(i)$, although they do preserve orthogonality for homogeneous vectors, do not preserve inner products.

\section{Goldman-Rota recurrence}

In this section we prove Theorem 1.4. As stated in the introduction, we identify $\mathbb{F}_{q}^{k}$, for $k<n$, with the subspace of $\mathbb{F}_{q}^{n}$ consisting of all vectors with the last $n-k$ components zero. We denote by $e_{1}, \ldots, e_{n}$ the standard basis vectors of $\mathbb{F}_{q}^{n}$. So $B_{q}(k)$ consists of all subspaces of $\mathbb{F}_{q}^{n}$ contained in the subspace spanned by $e_{1}, \ldots, e_{k}$.

Define $A_{q}(n)$ to be the collection of all subspaces in $B_{q}(n)$ not contained in the hyperplane $\mathbb{F}_{q}^{n-1}$, i.e.,

$$
A_{q}(n)=B_{q}(n)-B_{q}(n-1)=\left\{X \in B_{q}(n): X \nsubseteq \mathbb{F}_{q}^{n-1}\right\}, n \geqslant 1 .
$$

For $1 \leqslant k \leqslant n$, let $A_{q}(n)_{k}$ denote the set of all subspaces in $A_{q}(n)$ with dimension $k$. We consider $A_{q}(n)$ as an induced subposet of $B_{q}(n)$.

Define a map

$$
\mathcal{H}(n): A_{q}(n) \rightarrow B_{q}(n-1)
$$

by $\mathcal{H}(n)(X)=X \cap \mathbb{F}_{q}^{n-1}$, for $X \in A_{q}(n)$. Define an equivalence relation $\sim$ on $A_{q}(n)$ by $X \sim Y$ iff $\mathcal{H}(n)(X)=\mathcal{H}(n)(Y)$. Denote the equivalence class of $X \in A_{q}(n)$ by $[X]$.

For $X \in B_{q}(n-1)$, define $\widehat{X}$ to be the subspace in $A_{q}(n)$ spanned by $X$ and $e_{n}$.

Lemma 2.1. Let $X, Y \in A_{q}(n)$ and $Z, T \in B_{q}(n-1)$. Then

(i) $\operatorname{dim} \mathcal{H}(n)(X)=\operatorname{dim} X-1$ and $\widehat{\mathcal{H}(n)(X)} \in[X]$.

(ii) $Z \leqslant T$ iff $\widehat{Z} \leqslant \widehat{T}$. 
(iii) $Y$ covers $X$ iff $\mathcal{H}(n)(Y)$ covers $\mathcal{H}(n)(X)$ and $Y=\operatorname{span}(\mathcal{H}(n)(Y) \cup\{v\})$ for any $v \in X-\mathbb{F}_{q}^{n-1}$.

(iv) $\left|\mathcal{H}(n)^{-1}(Z)\right|=q^{l}$, where $l=n-\operatorname{dim} Z-1$. Thus, $|[X]|=q^{n-k}$, where $k=\operatorname{dim} X$.

Proof. (i), (ii), and (iii) are clear.

(iv) Let $\mathcal{H}(n)^{-1}(Z)=\left\{Y_{1}, \ldots, Y_{t}\right\}$. Then $Y_{i} \cap Y_{j}=Z, 1 \leqslant i \neq j \leqslant t$. Let $\operatorname{dim}(Z)=m$. Now $\left|Y_{i}-Z\right|=q^{m+1}-q^{m}$ for all $i$ and thus $t=\frac{q^{n}-q^{n-1}}{q^{m+1}-q^{m}}=q^{n-m-1}$.

We have an orthogonal decomposition

$$
V\left(B_{q}(n+1)\right)=V\left(B_{q}(n)\right) \oplus V\left(A_{q}(n+1)\right) .
$$

We shall now give a canonical orthogonal decomposition of $V\left(A_{q}(n+1)\right)$.

Let $H(n+1, q)$ denote the subgroup of $G L(n+1, q)=G L\left(n+1, \mathbb{F}_{q}\right)$ consisting of all matrices of the form

$$
\left[\begin{array}{cc} 
& a_{1} \\
I & \cdot \\
& \cdot \\
0 \cdots 0 & a_{n}
\end{array}\right]
$$

where $I$ is the $n \times n$ identity matrix.

The additive abelian group $\mathbb{F}_{q}^{n}$ is isomorphic to $H(n+1, q)$ via $\phi: \mathbb{F}_{q}^{n} \rightarrow H(n+1, q)$ given by

$$
\phi\left(\left[\begin{array}{c}
a_{1} \\
\cdot \\
\cdot \\
a_{n}
\end{array}\right]\right) \rightarrow\left[\begin{array}{cc} 
& a_{1} \\
I & \cdot \\
& \cdot \\
0 \cdots 0 & a_{n} \\
0 \cdots
\end{array}\right] .
$$

There is a natural (left) action of $H(n+1, q)$ on $A_{q}(n+1)$ and $A_{q}(n+1)_{k}$. For $X \in A_{q}(n+1)$, let $G_{X} \subseteq H(n+1, q)$ denote the stabilizer of $X$.

Lemma 2.2. Let $X, Y \in A_{q}(n+1)$. Then

(i) The orbit of $X$ under the action of $H(n+1, q)$ is $[X]$.

(ii) Suppose $Y$ covers $X$. Then the bipartite graph of the covering relations between $[Y]$ and $[X]$ is regular with degrees $q$ (on the $[Y]$ side) and 1 (on the $[X]$ side).

(iii) Suppose $X \subseteq Y$. Then $G_{X} \subseteq G_{Y}$.

Proof. (i) This is clear.

(ii) Since the action of $H(n+1, q)$ on $A_{q}(n+1)$ is clearly order preserving it follows that the bipartite graph in the statement is biregular (i.e., the degree is constant on each part). 
Let $Y^{\prime} \in[Y]$ also cover $X$. Then $\mathcal{H}(n+1)\left(Y^{\prime}\right)=\mathcal{H}(n+1)(Y)$ and it follows from Lemma 2.1(iii) that $Y=Y^{\prime}$. So the degree on the $[X]$ side is 1 . Let $\operatorname{dim}(X)=k$. Then, by Lemma 2.1(iv), $|[Y]|=q^{n-k}$ and $|[X]|=q^{n+1-k}$ and hence, by regularity, the degree on the $[Y]$ side is $q$.

(iii) We may assume that $Y$ covers $X$. If $X \neq X^{\prime} \in[X]$ then $X \cap X^{\prime}=\mathcal{H}(n+1)(X)$. So, by part (ii) and Lemma 2.1(iii), we can write $Y$ as a union

$$
Y=X_{1} \cup X_{2} \cup \cdots X_{q}
$$

of subspaces $X=X_{1}, \ldots, X_{q} \in[X]$ with $X_{i} \cap X_{j}=\mathcal{H}(n+1)(X), 1 \leqslant i \neq j \leqslant q$.

Now the stabilizer of all the elements $X_{1}, \ldots, X_{q}$ is $G_{X}$ (since $H(n+1, q)$ is commutative). It follows that $G_{X} \subseteq G_{Y}$.

Let $\mathcal{I}_{q}(n)$ denote the set of all distinct irreducible characters (all of degree 1$)$ of $H(n+$ $1, q)$ and let $\mathcal{N}_{q}(n)$ denote the set of all distinct nontrivial irreducible characters of $H(n+$ $1, q)$.

Let $\psi_{k}$ (respectively, $\psi$ ) denote the character of the permutation representation of $H(n+1, q)$ on $V\left(A_{q}(n+1)_{k}\right)$ (respectively, $\left.V\left(A_{q}(n+1)\right)\right)$ corresponding to the left action. Clearly $\psi=\sum_{k=1}^{n+1} \psi_{k}$. Below [,] denotes character inner product and the $q$-binomial coefficient $\left[\begin{array}{l}n \\ k\end{array}\right]_{q}$ is taken to be zero when $n$ or $k$ is $<0$.

Theorem 2.3. (i) For $1 \leqslant k \leqslant n+1$ and $g \in H(n+1, q)$ we have

$$
\psi_{k}(g)= \begin{cases}q^{n-k+1}\left[\begin{array}{c}
n \\
k-1
\end{array}\right]_{q} & \text { if } g=I, \\
q^{n-k+1}\left[\begin{array}{c}
n-1 \\
k-2
\end{array}\right]_{q} & \text { if } g \neq I .\end{cases}
$$

(ii) Let $\chi \in \mathcal{I}_{q}(n)$ be the trivial character. Then $\left[\chi, \psi_{k}\right]=\left[\begin{array}{c}n \\ k-1\end{array}\right]_{q}, 1 \leqslant k \leqslant n+1$.

(iii) Let $\chi \in \mathcal{N}_{q}(n)$. Then $\left[\chi, \psi_{k}\right]=\left[\begin{array}{c}n-1 \\ k-1\end{array}\right]_{q}, 1 \leqslant k \leqslant n+1$.

(iv)

$$
\left[\begin{array}{c}
n+1 \\
k
\end{array}\right]_{q}=\left[\begin{array}{l}
n \\
k
\end{array}\right]_{q}+\left[\begin{array}{c}
n \\
k-1
\end{array}\right]_{q}+\left(q^{n}-1\right)\left[\begin{array}{l}
n-1 \\
k-1
\end{array}\right]_{q}, n, k \geqslant 1
$$

with $\left[\begin{array}{l}0 \\ k\end{array}\right]_{q}=\delta(0, k)$ (Kronecker delta) and $\left[\begin{array}{l}n \\ 0\end{array}\right]_{q}=1$. Note that (4) follows by summing over $k$.

Proof. (i) From Lemma 2.1(iv),

$$
\left|A_{q}(n+1)_{k}\right|=q^{n-k+1}\left[\begin{array}{c}
n \\
k-1
\end{array}\right]_{q}=\psi_{k}(I) .
$$


Now assume $g \in H(n+1, q), g \neq I$ and let $X \in A_{q}(n+1)_{k}$. Let the last column of $g$ be $\left(a_{1}, \ldots, a_{n}, 1\right)^{t}(t=$ transpose $)$, where not all the $a_{i}$ 's are 0 . Now note that

(a) For $b_{1}, \ldots, b_{n} \in \mathbb{F}_{q}$,

$$
g\left(b_{1}, \ldots, b_{n}, 1\right)^{t}=\left(a_{1}+b_{1}, \ldots, a_{n}+b_{n}, 1\right)^{t} .
$$

(b) From item (a) above it follows that $g X=X$ iff the nonzero vector $\left(a_{1}, \ldots, a_{n}, 0\right)^{t} \in$ $\mathcal{H}(n+1)(X)$.

(c) From item (b) above, if $g X=X$ then $g Y=Y$ for all $Y \in[X]$. Thus $g$ either fixes all elements of $[X]$ or no elements.

(d) The number of subspaces in $B_{q}(n)_{k-1}$ containing the nonzero vector $\left(a_{1}, \ldots, a_{n}, 0\right)^{t}$ is $\left[\begin{array}{l}n-1 \\ k-2\end{array}\right]_{q}$.

It follows from items (b), (c), (d) above that $\psi_{k}(g)=q^{n-k+1}\left[\begin{array}{l}n-1 \\ k-2\end{array}\right]_{q}$.

(ii) This follows from the well known result that the multiplicity of the trivial representation in a permutation representation is the number of orbits, which in the present case is $\left[\begin{array}{c}n \\ k-1\end{array}\right]_{q}$.

(iii) Since $\chi$ is nontrivial we have $\sum_{g \in H(n+1, q)} \overline{\chi(g)}=0$ and thus $\sum_{g \in H(n+1, q), g \neq I} \overline{\chi(g)}=-1$.

Thus (below the sum is over all $g \neq I$ in $H(n+1, q)$ )

$$
\begin{aligned}
{\left[\chi, \psi_{k}\right] } & =\frac{1}{q^{n}}\left\{\psi_{k}(I)+\sum_{g \neq I} \overline{\chi(g)} \psi_{k}(g)\right\} \\
& =\frac{1}{q^{n}}\left\{q^{n-k+1}\left[\begin{array}{c}
n \\
k-1
\end{array}\right]_{q}-q^{n-k+1}\left[\begin{array}{l}
n-1 \\
k-2
\end{array}\right]_{q}\right\} \\
& =q^{-(k-1)}\left\{\left[\begin{array}{c}
n \\
k-1
\end{array}\right]_{q}-\left[\begin{array}{l}
n-1 \\
k-2
\end{array}\right]_{q}\right\} \\
& =\left[\begin{array}{l}
n-1 \\
k-1
\end{array}\right]_{q}
\end{aligned}
$$

where in the last step we have used $q$-Pascal's triangle (see Section 1.7 in [29])

$$
\left[\begin{array}{c}
n \\
k-1
\end{array}\right]_{q}=\left[\begin{array}{l}
n-1 \\
k-2
\end{array}\right]_{q}+q^{k-1}\left[\begin{array}{l}
n-1 \\
k-1
\end{array}\right]_{q} .
$$

(iv) Let $1 \leqslant k \leqslant n+1$. Restricting (10) to dimension $k$ we get the following orthogonal decomposition

$$
V\left(B_{q}(n+1)_{k}\right)=V\left(B_{q}(n)_{k}\right) \oplus V\left(A_{q}(n+1)_{k}\right)
$$


Splitting $V\left(A_{q}(n+1)_{k}\right)$ into $H(n+1, q)$-irreducibles and taking dimensions using parts (ii) and (iii) we get the result. The initial conditions are easily verified.

For $\chi \in \mathcal{I}_{q}(n)$, let $W(\chi)$ (respectively, $\left.W(\chi)_{k}\right)$ denote the isotypical component of $V\left(A_{q}(n+1)\right)$ (respectively, $\left.V\left(A_{q}(n+1)_{k}\right)\right)$ corresponding to the irreducible representation of $H(n+1, q)$ with character $\chi$. When $\chi$ is the trivial character we denote $W(\chi)$ (respectively, $\left.W(\chi)_{k}\right)$ by $W(0)$ (respectively, $\left.W(0)_{k}\right)$. Note that the standard inner product on $V\left(B_{q}(n+1)\right)$ is $G L(n+1, q)$-invariant (and thus, in particular $H(n+1, q)$-invariant). Thus, we have the following orthogonal decompositions, the last of which is canonical (note that $W(\chi)_{n+1}$, for $\chi \in \mathcal{N}_{q}(n)$, is the zero module, by Theorem 2.3(iii)).

$$
\begin{aligned}
W(0) & =W(0)_{1} \oplus \cdots \oplus W(0)_{n+1}, \\
W(\chi) & =W(\chi)_{1} \oplus \cdots \oplus W(\chi)_{n}, \quad \chi \in \mathcal{N}_{q}(n), \\
V\left(A_{q}(n+1)\right) & =W(0) \oplus\left(\oplus_{\chi \in \mathcal{N}_{q}(n)} W(\chi)\right) .
\end{aligned}
$$

Since $U_{n+1}$ is $G L(n+1, q)$-linear, each of $W(0)$ and $W(\chi), \chi \in \mathcal{N}_{q}(n)$ is $U_{n+1}$-closed.

For $\chi \in \mathcal{I}_{q}(n)$, define the following element of the group algebra of $H(n+1, q)$ :

$$
p(\chi)=\sum_{g} \overline{\chi(g)} g
$$

where the sum is over all $g \in H(n+1, q)$. For $1 \leqslant k \leqslant n+1$, the map

$$
p(\chi): V\left(A_{q}(n+1)_{k}\right) \rightarrow V\left(A_{q}(n+1)_{k}\right),
$$

given by $v \mapsto \sum_{g \in H(n+1, q)} \overline{\chi(g)} g v$, is a nonzero multiple of the $H(n+1, q)$-linear projection onto $W(\chi)_{k}$. Similarly for $p(\chi): V\left(A_{q}(n+1)\right) \rightarrow V\left(A_{q}(n+1)\right)$.

Lemma 2.4. Let $X \in A_{q}(n+1)$ and $\chi \in \mathcal{I}_{q}(n)$. Then $p(\chi)(X) \neq 0$ iff $\chi: G_{X} \rightarrow \mathbb{C}^{*}$ is the trivial character of $G_{X}$.

Proof Let $\left\{h_{0}=1, h_{1}, \ldots, h_{t}\right\}$ be a set of distinct coset representatives of $G_{X}$, i.e.,

$$
H(n+1, q)=G_{X} h_{0} \cup G_{X} h_{1} \cup \cdots \cup G_{X} h_{t} \quad \text { (disjoint union). }
$$

Write $[X]=\left\{X=X_{0}, X_{1}, \ldots, X_{t}\right\}$ and assume without loss of generality that $h_{i} X=$ $X_{i}, 0 \leqslant i \leqslant t$. Note that $G_{X}$ is the stabilizer of all the elements of $[X]$.

We have

$$
\begin{aligned}
p(\chi)(X) & =\sum_{g \in H(n+1, q)} \overline{\chi(g)} g X \\
& =\left(\sum_{g \in G_{X}} \overline{\chi(g)}\right) X+\sum_{i=1}^{t} \overline{\chi\left(h_{i}\right)}\left(\sum_{g \in G_{X}} \overline{\chi(g)}\right) X_{i} .
\end{aligned}
$$

The result follows since $\sum_{g \in G_{X}} \overline{\chi(g)}=0$ for every nontrivial character of $G_{X}$. 
Theorem 2.5. (i) Let $\chi \in \mathcal{I}_{q}(n), X, Y \in A_{q}(n+1)$ with $X \sim Y$. Then $p(\chi)(X)$ is a nonzero multiple of $p(\chi)(Y)$.

(ii) Let $\chi \in \mathcal{I}_{q}(n)$. Then $\left\{p(\chi)(\widehat{X}): X \in B_{q}(n)_{k-1}\right.$ with $\left.p(\chi)(\widehat{X}) \neq 0\right\}$ is a basis of $W(\chi)_{k}, 1 \leqslant k \leqslant n+1$.

(iii) Let $\chi \in \mathcal{I}_{q}(n)$ and let $X, Y \in B_{q}(n)$ with $X$ covering $Y$. Then

$$
p(\chi)(\widehat{X}) \neq 0 \text { implies } p(\chi)(\widehat{Y}) \neq 0 .
$$

(iv) Define $\theta_{n}: V\left(B_{q}(n)\right) \rightarrow W(0)$ by

$$
X \mapsto \sum_{Y \sim \widehat{X}} Y, X \in B_{q}(n) .
$$

Then $\theta_{n}$ is an isomorphism of pairs $\left(V\left(B_{q}(n)\right), q U_{n}\right)$ and $\left(W(0), U_{n+1}\right)$ and

$$
\begin{aligned}
U_{n+1}(v) & =U_{n}(v)+\theta_{n}(v), v \in V\left(B_{q}(n)\right), \\
\left\langle\theta_{n}(w), \theta_{n}(v)\right\rangle & =q^{n-k}\langle w, v\rangle, w, v \in V\left(B_{q}(n)_{k}\right), 0 \leqslant k \leqslant n .
\end{aligned}
$$

(v) Let $\chi \in \mathcal{N}_{q}(n)$. From Theorem 2.3 (iii) we have $\operatorname{dim} W(\chi)_{n}=1$. It thus follows from part (ii) that there is a unique element $X=X(\chi) \in B_{q}(n)_{n-1}$ such that $p(\chi)(\widehat{X}) \neq 0$. Define $\lambda(\chi): V\left(B_{q}(X)\right) \rightarrow W(\chi)$ by

$$
Y \mapsto p(\chi)(\widehat{Y}), Y \in B_{q}(X) .
$$

Then $\lambda(\chi)$ is an isomorphism of pairs $\left(V\left(B_{q}(X)\right), U_{X}\right)$ and $\left(W(\chi), U_{n+1}\right)$ and satisfies

$$
\langle\lambda(\chi)(w), \lambda(\chi)(v)\rangle=q^{n+k}\langle w, v\rangle, w, v \in V\left(B_{q}(X)_{k}\right), 0 \leqslant k \leqslant n-1 .
$$

(vi) For $X \in B_{q}(n)_{n-1}$ the number of $\chi \in \mathcal{N}_{q}(n)$ such that $p(\chi)(\widehat{X}) \neq 0$ is $q-1$.

Proof. (i) By Lemma 2.2(i), $X=h Y$ for some $h \in H(n+1, q)$. Then we have (below the sum is over all $g \in H(n+1, q))$

$$
p(\chi)(X)=\sum_{g} \overline{\chi(g)} g h Y=\sum_{g} \overline{\chi\left(g h^{-1}\right)} g Y=\overline{\chi\left(h^{-1}\right)} p(\chi)(Y) .
$$

Since $\chi\left(h^{-1}\right) \neq 0(\chi$ being of degree 1$)$ the result follows.

(ii) The map (16) is a projection onto $W(\chi)_{k}$, so $\mathcal{G}=\left\{p(\chi)(X): X \in A_{q}(n+1)_{k}\right\}$ spans $W(\chi)_{k}$. By part (i), the subset $\mathcal{G}^{\prime}=\left\{p(\chi)(\widehat{X}): X \in B_{q}(n)_{k-1}\right.$ with $\left.p(\chi)(\widehat{X}) \neq 0\right\}$ also spans $W(\chi)_{k}$. Now, for distinct $X, Y \in B_{q}(n)_{k-1}, p(\chi)(\widehat{X})$ and $p(\chi)(\widehat{Y})$ have disjoint supports, so $\mathcal{G}^{\prime}$ is a basis.

(iii) This follows from Lemma 2.2(iii) and Lemma 2.4. 
(iv) By Theorem 2.3 (ii) the dimensions of $V\left(B_{q}(n)\right)$ and $W(0)$ are the same. For $X_{1} \neq$ $X_{2} \in B_{q}(n)$ the supports of $\theta_{n}\left(X_{1}\right)$ and $\theta_{n}\left(X_{2}\right)$ are disjoint. It follows that $\theta_{n}$ is a vector space isomorphism. Clearly, (18) holds.

Let $X \in B_{q}(n)$ with $\operatorname{dim} X=k$. Then $|[\widehat{X}]|=q^{n-k}$ by Lemma 2.1(iv), showing (19).

We have (below the sum is over all $Z$ covering $X$ in $B_{q}(n)$ )

$$
\begin{aligned}
\theta_{n}\left(q U_{n}(X)\right) & =\theta_{n}\left(q\left(\sum_{Z} Z\right)\right) \\
& =q\left\{\sum_{Z} \sum_{Y \sim \widehat{Z}} Y\right\} .
\end{aligned}
$$

Similarly (in the second step below $T$ varies over all subspaces covering $Y$ and in the third step $Z$ varies over all subspaces in $B_{q}(n)$ covering $X$. We have used Lemma 2.1(ii) and Lemma 2.2(ii) to go from the second to the third step)

$$
\begin{aligned}
U_{n+1}\left(\theta_{n}(X)\right) & =U_{n+1}\left(\sum_{Y \sim \widehat{X}} Y\right) \\
& =\sum_{Y \sim \widehat{X}} \sum_{T} T \\
& =q\left\{\sum_{Z} \sum_{Y \sim \widehat{Z}} Y\right\} .
\end{aligned}
$$

(v) By part (iii) it follows that $\lambda(\chi)(Y) \neq 0$ for all $Y \in B_{q}(X)$. By Theorem 2.3 (iii) the dimensions of $V\left(B_{q}(X)\right)$ and $W(\chi)$ are the same. For $Y_{1} \neq Y_{2} \in B_{q}(X)$ the supports of $\lambda(\chi)\left(Y_{1}\right)$ and $\lambda(\chi)\left(Y_{2}\right)$ are disjoint. It follows that $\lambda(\chi)$ is a vector space isomorphism.

Now, for $Y \in B_{q}(X)$, we have (below the sum is over all $Z$ covering $Y$ in $B_{q}(X)$ )

$$
\begin{aligned}
\lambda(\chi)\left(U_{X}(Y)\right) & =\lambda(\chi)\left(\sum_{Z} Z\right) \\
& =\sum_{Z} p(\chi)(\widehat{Z}) .
\end{aligned}
$$

Let $Y \in B_{q}(X)$. Before calculating $U_{n+1} \lambda(\chi)(Y)$ we make the following observation. By Lemma 2.1(ii) every element covering $\widehat{Y}$ is of the form $\widehat{Z}$, for some $Z$ covering $Y$ in $B_{q}(n)$. Suppose $Z \in B_{q}(n)-B_{q}(X)$. Since $\operatorname{dim} W(\chi)=G_{q}(n-1)$ (by Theorem 2.3(iii)), it follows by parts (ii) and (iii) that $p(\chi)(\widehat{Z})=0$.

We now calculate $U_{n+1} \lambda(\chi)(Y)$. In the second step below we have used the fact that $U_{n+1}$ is $H(n+1, q)$-linear and in the third step, using the observation in the paragraph above, we may restrict the sum to all $Z$ covering $Y$ in $B_{q}(X)$. 
We have

$$
\begin{aligned}
U_{n+1}(\lambda(\chi)(Y)) & =U_{n+1}(p(\chi)(\widehat{Y})) \\
& =p(\chi)\left(U_{n+1}(\widehat{Y})\right) \\
& =\sum_{Z} p(\chi)(\widehat{Z})
\end{aligned}
$$

We will now show that $\|p(\chi)(\widehat{Y})\|=\sqrt{q^{n+k}}$ if $Y \in B_{q}(X)$ with $\operatorname{dim} Y=k$. This will prove $(20)$.

By Lemma 2.1(iv), $|[\widehat{Y}]|=q^{n+1-(k+1)}=q^{n-k}$ and so $\left|G_{\widehat{Y}}\right|=q^{k}$. It now follows from (17) (since the restriction of $\chi$ to $G_{\widehat{Y}}$ must be trivial) that $|p(\chi)(\widehat{Y})|=\sqrt{q^{n-k}\left(q^{k}\right)^{2}}=$ $\sqrt{q^{n+k}}$.

(vi) By Theorem 2.3(iii), $\sum_{\chi \in \mathcal{N}_{q}(n)} \operatorname{dim} W(\chi)_{n}=q^{n}-1=(q-1)\left(1+q+\cdots+q^{n-1}\right)$. Now $\left|B_{q}(n)_{n-1}\right|=\left[\begin{array}{c}n \\ n-1\end{array}\right]_{q}=1+q+\cdots+q^{n-1}$ and by Lemma 2.1(iv), $|[\widehat{X}]|=q$ for $X \in B_{q}(n)_{n-1}$. Since, for $\chi \in \mathcal{N}_{q}(n)$ and $X \in B_{q}(n)_{n-1}$, the support of $p(\chi)(\widehat{X})$ is contained in $[\widehat{X}]$ and $p(\chi)(\widehat{X})$ is orthogonal to $p(\pi)(\widehat{X})$ (where $\pi$ is the trivial character), the result now follows by part (ii).

To use Theorem 2.5 for computations we need the character table of $H(n+1, q)$, which is easy to write down explicitly since $H(n+1, q)$ is a direct sum of $n$ cyclic groups of order $q$. We now give a small example to illustrate part (v) of Theorem 2.5.

Example Let $q=3, n=2$, and $\omega=e^{2 \pi \mathrm{i} / 3}$. Consider $A_{3}(3)$ with the $H(3,3)$-action. We write the elements of $\mathbb{F}_{3}$ as $\{0,1,2\}$ and define $\chi \in \mathcal{N}_{3}(2)$ by $\chi\left(\phi\left(\left(a_{1}, a_{2}\right)\right)=\omega^{2 a_{1}+a_{2}}\right.$, where $2 a_{1}+a_{2}$ is computed as an integer.

We have

$$
\begin{aligned}
p(\chi)= & {\left[\begin{array}{lll}
1 & 0 & 0 \\
0 & 1 & 0 \\
0 & 0 & 1
\end{array}\right]+\omega^{2}\left[\begin{array}{lll}
1 & 0 & 0 \\
0 & 1 & 1 \\
0 & 0 & 1
\end{array}\right]+\omega\left[\begin{array}{lll}
1 & 0 & 0 \\
0 & 1 & 2 \\
0 & 0 & 1
\end{array}\right] } \\
& +\omega\left[\begin{array}{lll}
1 & 0 & 1 \\
0 & 1 & 0 \\
0 & 0 & 1
\end{array}\right]+\left[\begin{array}{lll}
1 & 0 & 1 \\
0 & 1 & 1 \\
0 & 0 & 1
\end{array}\right]+\omega^{2}\left[\begin{array}{lll}
1 & 0 & 1 \\
0 & 1 & 2 \\
0 & 0 & 1
\end{array}\right] \\
& +\omega^{2}\left[\begin{array}{lll}
1 & 0 & 2 \\
0 & 1 & 0 \\
0 & 0 & 1
\end{array}\right]+\omega\left[\begin{array}{lll}
1 & 0 & 2 \\
0 & 1 & 1 \\
0 & 0 & 1
\end{array}\right]+\left[\begin{array}{lll}
1 & 0 & 2 \\
0 & 1 & 2 \\
0 & 0 & 1
\end{array}\right]
\end{aligned}
$$

For a finite set of vectors $v_{1}, \ldots, v_{m}$, we shall denote by $\mathcal{S}\left(v_{1}, \ldots, v_{m}\right)$ the subspace spanned by them. 
The four subspaces in $B_{3}(2)_{1}$ are

$$
X_{1}=\mathcal{S}\left(\left[\begin{array}{l}
1 \\
0 \\
0
\end{array}\right]\right), X_{2}=\mathcal{S}\left(\left[\begin{array}{l}
0 \\
1 \\
0
\end{array}\right]\right), X_{3}=\mathcal{S}\left(\left[\begin{array}{l}
1 \\
1 \\
0
\end{array}\right]\right), X_{4}=\mathcal{S}\left(\left[\begin{array}{l}
2 \\
1 \\
0
\end{array}\right]\right) \text {. }
$$

It can be checked that

$$
\begin{aligned}
& p(\chi)\left(\widehat{X_{1}}\right)= \\
& \quad \mathcal{S}\left(\left[\begin{array}{l}
1 \\
0 \\
0
\end{array}\right],\left[\begin{array}{l}
0 \\
0 \\
1
\end{array}\right]\right)+\omega^{2} \mathcal{S}\left(\left[\begin{array}{l}
1 \\
0 \\
0
\end{array}\right],\left[\begin{array}{l}
0 \\
1 \\
1
\end{array}\right]\right)+\omega \mathcal{S}\left(\left[\begin{array}{l}
1 \\
0 \\
0
\end{array}\right],\left[\begin{array}{l}
0 \\
2 \\
1
\end{array}\right]\right) \\
& \quad+\omega \mathcal{S}\left(\left[\begin{array}{l}
1 \\
0 \\
0
\end{array}\right],\left[\begin{array}{l}
1 \\
0 \\
1
\end{array}\right]\right)+\mathcal{S}\left(\left[\begin{array}{l}
1 \\
0 \\
0
\end{array}\right],\left[\begin{array}{l}
1 \\
1 \\
1
\end{array}\right]\right)+\omega^{2} \mathcal{S}\left(\left[\begin{array}{l}
1 \\
0 \\
0
\end{array}\right],\left[\begin{array}{l}
1 \\
2 \\
1
\end{array}\right]\right) \\
& \quad+\omega^{2} \mathcal{S}\left(\left[\begin{array}{l}
1 \\
0 \\
0
\end{array}\right],\left[\begin{array}{l}
2 \\
0 \\
1
\end{array}\right]\right)+\omega \mathcal{S}\left(\left[\begin{array}{l}
1 \\
0 \\
0
\end{array}\right],\left[\begin{array}{l}
2 \\
1 \\
1
\end{array}\right]\right)+\mathcal{S}\left(\left[\begin{array}{l}
1 \\
0 \\
0
\end{array}\right],\left[\begin{array}{l}
2 \\
2 \\
1
\end{array}\right]\right) .
\end{aligned}
$$

Using $\omega^{3}=1$ and $1+\omega+\omega^{2}=0$ we see that $p\left(\chi\left(\widehat{X_{1}}\right)\right)=0$.

Similarly we can check that

$$
\begin{aligned}
& p(\chi)\left(\widehat{X_{2}}\right)= \\
& \quad \mathcal{S}\left(\left[\begin{array}{l}
0 \\
1 \\
0
\end{array}\right],\left[\begin{array}{l}
0 \\
0 \\
1
\end{array}\right]\right)+\omega^{2} \mathcal{S}\left(\left[\begin{array}{l}
0 \\
1 \\
0
\end{array}\right],\left[\begin{array}{l}
0 \\
1 \\
1
\end{array}\right]\right)+\omega \mathcal{S}\left(\left[\begin{array}{l}
0 \\
1 \\
0
\end{array}\right],\left[\begin{array}{l}
0 \\
2 \\
1
\end{array}\right]\right) \\
& \quad+\omega \mathcal{S}\left(\left[\begin{array}{l}
0 \\
1 \\
0
\end{array}\right],\left[\begin{array}{l}
1 \\
0 \\
1
\end{array}\right]\right)+\mathcal{S}\left(\left[\begin{array}{l}
0 \\
1 \\
0
\end{array}\right],\left[\begin{array}{l}
1 \\
1 \\
1
\end{array}\right]\right)+\omega^{2} \mathcal{S}\left(\left[\begin{array}{l}
0 \\
1 \\
0
\end{array}\right],\left[\begin{array}{l}
1 \\
2 \\
1
\end{array}\right]\right) \\
& \quad+\omega^{2} \mathcal{S}\left(\left[\begin{array}{l}
0 \\
1 \\
0
\end{array}\right],\left[\begin{array}{l}
2 \\
0 \\
1
\end{array}\right]\right)+\omega \mathcal{S}\left(\left[\begin{array}{l}
0 \\
1 \\
0
\end{array}\right],\left[\begin{array}{l}
2 \\
1 \\
1
\end{array}\right]\right)+\mathcal{S}\left(\left[\begin{array}{l}
0 \\
1 \\
0
\end{array}\right],\left[\begin{array}{l}
2 \\
2 \\
1
\end{array}\right]\right),
\end{aligned}
$$

and

$$
\begin{aligned}
& p(\chi)\left(\widehat{X_{4}}\right)= \\
& \quad \mathcal{S}\left(\left[\begin{array}{l}
2 \\
1 \\
0
\end{array}\right],\left[\begin{array}{l}
0 \\
0 \\
1
\end{array}\right]\right)+\omega^{2} \mathcal{S}\left(\left[\begin{array}{l}
2 \\
1 \\
0
\end{array}\right],\left[\begin{array}{l}
0 \\
1 \\
1
\end{array}\right]\right)+\omega \mathcal{S}\left(\left[\begin{array}{l}
2 \\
1 \\
0
\end{array}\right],\left[\begin{array}{l}
0 \\
2 \\
1
\end{array}\right]\right) \\
& \quad+\omega \mathcal{S}\left(\left[\begin{array}{l}
2 \\
1 \\
0
\end{array}\right],\left[\begin{array}{l}
1 \\
0 \\
1
\end{array}\right]\right)+\mathcal{S}\left(\left[\begin{array}{l}
2 \\
1 \\
0
\end{array}\right],\left[\begin{array}{l}
1 \\
1 \\
1
\end{array}\right]\right)+\omega^{2} \mathcal{S}\left(\left[\begin{array}{l}
2 \\
1 \\
0
\end{array}\right],\left[\begin{array}{l}
1 \\
2 \\
1
\end{array}\right]\right)
\end{aligned}
$$




$$
+\omega^{2} \mathcal{S}\left(\left[\begin{array}{l}
2 \\
1 \\
0
\end{array}\right],\left[\begin{array}{l}
2 \\
0 \\
1
\end{array}\right]\right)+\omega \mathcal{S}\left(\left[\begin{array}{l}
2 \\
1 \\
0
\end{array}\right],\left[\begin{array}{l}
2 \\
1 \\
1
\end{array}\right]\right)+\mathcal{S}\left(\left[\begin{array}{l}
2 \\
1 \\
0
\end{array}\right],\left[\begin{array}{l}
2 \\
2 \\
1
\end{array}\right]\right)
$$

are both equal to 0 and

$$
\begin{aligned}
& p(\chi)\left(\widehat{X_{3}}\right)= \\
& \quad \mathcal{S}\left(\left[\begin{array}{l}
1 \\
1 \\
0
\end{array}\right],\left[\begin{array}{l}
0 \\
0 \\
1
\end{array}\right]\right)+\omega^{2} \mathcal{S}\left(\left[\begin{array}{l}
1 \\
1 \\
0
\end{array}\right],\left[\begin{array}{l}
0 \\
1 \\
1
\end{array}\right]\right)+\omega \mathcal{S}\left(\left[\begin{array}{l}
1 \\
1 \\
0
\end{array}\right],\left[\begin{array}{l}
0 \\
2 \\
1
\end{array}\right]\right) \\
& +\omega \mathcal{S}\left(\left[\begin{array}{l}
1 \\
1 \\
0
\end{array}\right],\left[\begin{array}{l}
1 \\
0 \\
1
\end{array}\right]\right)+\mathcal{S}\left(\left[\begin{array}{l}
1 \\
1 \\
0
\end{array}\right],\left[\begin{array}{l}
1 \\
1 \\
1
\end{array}\right]\right)+\omega^{2} \mathcal{S}\left(\left[\begin{array}{l}
1 \\
1 \\
0
\end{array}\right],\left[\begin{array}{l}
1 \\
2 \\
1
\end{array}\right]\right) \\
& +\omega^{2} \mathcal{S}\left(\left[\begin{array}{l}
1 \\
1 \\
0
\end{array}\right],\left[\begin{array}{l}
2 \\
0 \\
1
\end{array}\right]\right)+\omega \mathcal{S}\left(\left[\begin{array}{l}
1 \\
1 \\
0
\end{array}\right],\left[\begin{array}{l}
2 \\
1 \\
1
\end{array}\right]\right)+\mathcal{S}\left(\left[\begin{array}{l}
1 \\
1 \\
0
\end{array}\right],\left[\begin{array}{l}
2 \\
2 \\
1
\end{array}\right]\right)
\end{aligned}
$$

is $\neq 0$.

We have now proved most of Theorem 1.4 except for one small detail. Let $X \in$ $B_{q}(n)_{n-1}$. The pairs $\left(V\left(B_{q}(X)\right), U_{X}\right)$ and $\left(V\left(B_{q}(n-1)\right), U_{n-1}\right)$ are clearly isomorphic with many possible isomorphisms. We now define a canonical isomorphism, based on the concept of a matrix in Schubert normal form.

A $n \times k$ matrix $M$ over $\mathbb{F}_{q}$ is in Schubert normal form (or, column reduced echelon form) provided

(i) Every column is nonzero.

(ii) The first nonzero entry in every column is a 1. Let the first nonzero entry in column $j$ occur in row $r_{j}$.

(iii) We have $r_{1}<r_{2}<\cdots<r_{k}$ and the submatrix of $M$ formed by the rows $r_{1}, r_{2}, \ldots, r_{k}$ is the $k \times k$ identity matrix.

It is well known that every $k$ dimensional subspace of $\mathbb{F}_{q}^{n}$ is the column space of a unique $n \times k$ matrix in Schubert normal form (see [29, Proposition 1.7.3] where the discussion is in terms of the row space).

Let $X \in B_{q}(n)_{n-1}$ and let $M(X)$ be the $n \times(n-1)$ matrix in Schubert normal form with column space $X$. The map $\tau(X): \mathbb{F}_{q}^{n-1} \rightarrow X$ given by $e_{j} \mapsto$ column $j$ of $M(X)$ is clearly a linear isomorphism and this isomorphism gives rise to an isomorphism

$$
\mu(X): V\left(B_{q}(n-1)\right) \rightarrow V\left(B_{q}(X)\right)
$$

of pairs $\left(V\left(B_{q}(n-1)\right), U_{n-1}\right)$ and $\left(V\left(B_{q}(X)\right), U_{X}\right)$ given by $\mu(X)(Y)=\tau(X)(Y), Y \in$ $B_{q}(n-1)$. 
Proof of Theorem 1.4. It is convenient to write the orthogonal decomposition (6) as follows

$$
V\left(B_{q}(n+1)\right)=V\left(B_{q}(n)\right) \oplus W(0) \oplus\left(\oplus_{\chi \in \mathcal{N}_{q}(n)} W(\chi)\right) .
$$

Note that $\left|\mathcal{N}_{q}(n)\right|=q^{n}-1$.

(i) We have already shown that each of $W(0)$ and $W(\chi), \chi \in \mathcal{N}_{q}(n)$ is $U_{n+1}$-closed. The rank sets of $W(0)$ and $W(\chi)$ are also easily seen to be as stated.

(ii) This follows from Theorem 2.5(iv).

(iii) Let $\chi \in \mathcal{N}_{q}(n)$. From Theorem 2.5(v) there is a unique $X \in B_{q}(n)_{n-1}$ with $p(\chi)(\widehat{X}) \neq$ 0 . It now follows, again by Theorem $2.5(\mathrm{v})$, that $\gamma_{n-1}(\chi)=\lambda(\chi) \mu(X)$ is an isomorphism of pairs $\left(V\left(B_{q}(n-1)\right), U_{n-1}\right)$ and $\left(W(\chi), U_{n+1}\right)$ satisfying $(9)$.

\section{Orthogonal symmetric Jordan basis and eigenvec- tors for the Grassmann scheme}

In this section we prove Theorem 1.3.

Proof (of Theorem 1.3) The proof is by induction on $n$, the result being clear for $n=0,1$.

Let $\chi \in \mathcal{N}_{q}(n)$ and let $\left(x_{k}, \ldots, x_{n-1-k}\right)$ be a SJC in $J_{q}(n-1)$ starting at rank $k$ and ending at rank $n-1-k$. Then, by Theorem 1.4, applied to the decomposition (21), $\left(y_{k+1}, \ldots, y_{n-k}\right)$, where $y_{u+1}=\gamma_{n-1}(\chi)\left(x_{u}\right), k \leqslant u \leqslant n-1-k$, is a SJC in $W(\chi)$ (with respect to $\left.U_{n+1}\right)$ starting at rank $k+1$ and ending at rank $n-k$. By (9) and the induction hypothesis we have, for $k+1 \leqslant u \leqslant n-k$,

$$
\frac{\left\|y_{u+1}\right\|}{\left\|y_{u}\right\|}=\frac{\sqrt{q^{n+u}}\left\|x_{u}\right\|}{\sqrt{q^{n+u-1}}\left\|x_{u-1}\right\|}=\sqrt{q^{k+1}[u+1-(k+1)]_{q}[n-k-u]_{q}} .
$$

Doing the procedure above for every SJC in $J_{q}(n-1)$ we get an orthogonal SJB of $W(\chi)$ satisfying (3). Note that, by definition of $\lambda(\chi)$, if the coefficients (in the standard basis) of the vectors in $J_{q}(n-1)$ were integral multiples of $q$ th roots of unity then so will be the coefficients of the vectors in the SJB of $W(\chi)$. Similarly, doing the procedure above for every $\chi \in \mathcal{N}_{q}(n)$ we get an orthogonal SJB, with respect to $U_{n+1}$, of $\oplus_{\chi \in \mathcal{N}_{q}(n)} W(\chi)$ satisfying (3).

Now we consider the subspace $V\left(B_{q}(n)\right) \oplus W(0)$. Let $\left(x_{k}, \ldots, x_{n-k}\right)$ be a SJC in $J_{q}(n)$, starting at rank $k$ and ending at rank $n-k$, and satisfying (3). Set $\overline{x_{u}}=\theta_{n}\left(x_{u}\right), k \leqslant u \leqslant$ $n-k$. Then, by Theorem 1.4, $\left(w_{k+1}, \ldots, w_{n-k+1}\right)$, where $w_{u+1}=q^{u-k} \overline{x_{u}}, k \leqslant u \leqslant n-k$ is a graded Jordan chain in $W(0)$ (with respect to $U_{n+1}$ ), starting at rank $k+1$ and ending at rank $n-k+1$. We have $U_{n+1}\left(q^{u-k} \overline{x_{u}}\right)=q^{u+1-k} \overline{x_{u+1}}$ and so

$$
U_{n+1}\left(\overline{x_{u}}\right)=q \overline{x_{u+1}}, k \leqslant u<n-k .
$$


Also we have, by (8),

$$
\left\langle\overline{x_{u}}, \overline{x_{u}}\right\rangle=q^{n-u}\left\langle x_{u}, x_{u}\right\rangle, k \leqslant u \leqslant n-k .
$$

For convenience we define $x_{k-1}=\overline{x_{k-1}}=x_{n+1-k}=0$. Note that (23) also holds for $u=k-1$.

Now, by (7), we have, for $k \leqslant u \leqslant n-k$,

$$
U_{n+1}\left(x_{u}\right)=x_{u+1}+\theta_{n}\left(x_{u}\right)=x_{u+1}+\overline{x_{u}}
$$

Let $Z$ be the subspace spanned by $\left\{x_{k}, \ldots, x_{n-k}\right\}$ and $\left\{\overline{x_{k}}, \ldots, \overline{x_{n-k}}\right\}$. Clearly, by (22) and (24), $Z$ is $U_{n+1}$-closed. We shall now get an orthogonal SJB of $Z$ satisfying (3) by taking linear combinations of the vectors $\left\{x_{k}, \ldots, x_{n-k}\right\}$ and $\left\{\overline{x_{k}}, \ldots, \overline{x_{n-k}}\right\}$.

We consider two cases:

(a) $k=n-k: \operatorname{By}(24)$,

$$
\left(x_{k}, \overline{x_{k}}\right)
$$

is an orthogonal SJB of $Z$ going from rank $k$ to rank $k+1$. We have, from (23), that $\frac{\left\langle\overline{x_{k}}, \overline{x_{k}}\right\rangle}{\left\langle x_{k}, x_{k}\right\rangle}=q^{k}$, and thus (3) is satisfied.

(b) $k<n-k$ : Define the following vectors in $Z$.

$$
\begin{aligned}
& y_{l}=x_{l}+[l-k]_{q} \overline{x_{l-1}}, \quad k \leqslant l \leqslant n+1-k, \\
& z_{l}=-q^{n} x_{l}+q^{l+k-1}[n-l-k+1]_{q} \overline{x_{l-1}}, \quad k+1 \leqslant l \leqslant n-k .
\end{aligned}
$$

Note that, using the induction hypothesis, the coefficients of $y_{l}, z_{l}$ are also integral multiples of $q$ th roots of unity. We claim that $\left(y_{k}, \ldots, y_{n+1-k}\right)$ and $\left(z_{k+1}, \ldots, z_{n-k}\right)$ form an orthogonal SJB of $Z$ satisfying (3).

We check orthogonality first, for which we need to show that $\left\langle y_{l}, z_{l}\right\rangle=0$ for $k+1 \leqslant$ $l \leqslant n-k$. Clearly $\left\langle x_{l}, \overline{x_{l-1}}\right\rangle=0$ for $k+1 \leqslant l \leqslant n-k$. Thus

$$
\left\langle y_{l}, z_{l}\right\rangle=-q^{n}\left\langle x_{l}, x_{l}\right\rangle+q^{k+l-1}[l-k]_{q}[n-l-k+1]_{q}\left\langle\overline{x_{l-1}}, \overline{x_{l-1}}\right\rangle .
$$

By the induction hypothesis $\left\langle x_{l}, x_{l}\right\rangle=q^{k}[l-k]_{q}[n-l-k+1]_{q}\left\langle x_{l-1}, x_{l-1}\right\rangle$ and by (23) $\left\langle\overline{x_{l-1}}, \overline{x_{l-1}}\right\rangle=q^{n+1-l}\left\langle x_{l-1}, x_{l-1}\right\rangle$. Thus $\left\langle y_{l}, z_{l}\right\rangle=0$.

Now we check the Jordan chain condition. Using (22) and (24), we have, for $k \leqslant l<$ $n+1-k$,

$$
\begin{aligned}
U_{n+1}\left(y_{l}\right) & =x_{l+1}+\left(1+q[l-k]_{q}\right) \overline{x_{l}} \\
& =x_{l+1}+[l+1-k]_{q} \overline{x_{l}} \\
& =y_{l+1}
\end{aligned}
$$

and clearly $U_{n+1}\left(y_{n+1-k}\right)=0$. 
Similarly, for $k+1 \leqslant l \leqslant n-k$,

$$
\begin{aligned}
U_{n+1}\left(z_{l}\right) & =-q^{n} x_{l+1}+\left(q^{l+k}[n-l-k+1]_{q}-q^{n}\right) \overline{x_{l}} \\
& =-q^{n} x_{l+1}+q^{l+k}[n-l-k]_{q} \overline{x_{l}} \\
& =z_{l+1} .
\end{aligned}
$$

Note that $z_{n-k+1}=0$.

Now we check that condition (3) holds. For $k \leqslant u<n+1-k$ we have by the induction hypothesis (in the second step below we have used (23). Note the second term in the denominator after the fourth step below. This is a fraction with a term $[u-k]_{q}$ in the denominator, which is zero for $u=k$. This is permissible here because of the presence of the factor $[u-k]_{q}^{2}$ in the numerator)

$$
\begin{aligned}
\frac{\left\langle y_{u+1}, y_{u+1}\right\rangle}{\left\langle y_{u}, y_{u}\right\rangle} & =\frac{\left\langle x_{u+1}, x_{u+1}\right\rangle+[u+1-k]_{q}{ }^{2}\left\langle\overline{x_{u}}, \overline{x_{u}}\right\rangle}{\left\langle x_{u}, x_{u}\right\rangle+[u-k]_{q}^{2}\left\langle\overline{x_{u-1}}, \overline{x_{u-1}}\right\rangle} \\
& =\frac{\left\langle x_{u+1}, x_{u+1}\right\rangle+q^{n-u}[u+1-k]_{q}{ }^{2}\left\langle x_{u}, x_{u}\right\rangle}{\left\langle x_{u}, x_{u}\right\rangle+q^{n-u+1}[u-k]_{q}{ }^{2}\left\langle x_{u-1}, x_{u-1}\right\rangle} \\
& =\frac{\frac{\left\langle x_{u+1}, x_{u+1}\right\rangle}{\left\langle x_{u}, x_{u}\right\rangle}+q^{n-u}[u+1-k]_{q}{ }^{2}}{1+q^{n-u+1}[u-k]_{q}{ }^{2} \frac{\left\langle x_{u-1}, x_{u-1}\right\rangle}{\left\langle x_{u}, x_{u}\right\rangle}} \\
& =\frac{q^{k}[u+1-k]_{q}[n-k-u]_{q}+q^{n-u}[u+1-k]_{q}{ }^{2}}{1+\frac{q^{n-u+1}[u-k]_{q}{ }^{2}}{q^{k}[u-k]_{q}[n-k-u+1]_{q}}} \\
& =q^{k}[u+1-k]_{q}[n-k-u+1]_{q}\left(\frac{[n-k-u]_{q}+q^{n-k-u}[u+1-k]_{q}}{[n-k-u+1]_{q}+q^{n-k-u+1}[u-k]_{q}}\right) \\
& =q^{k}[u+1-k]_{q}[n-k-u+1]_{q} .
\end{aligned}
$$

Similarly, for $k+1 \leqslant u<n-k$, we have

$$
\begin{aligned}
\frac{\left\langle z_{u+1}, z_{u+1}\right\rangle}{\left\langle z_{u}, z_{u}\right\rangle} & =\frac{q^{2 n}\left\langle x_{u+1}, x_{u+1}\right\rangle+q^{2 u+2 k}[n-u-k]_{q}{ }^{2}\left\langle\overline{x_{u}}, \overline{x_{u}}\right\rangle}{q^{2 n}\left\langle x_{u}, x_{u}\right\rangle+q^{2 u+2 k-2}[n-u-k+1]_{q}{ }^{2}\left\langle\overline{x_{u-1}}, \overline{x_{u-1}}\right\rangle} \\
& =\frac{\left\langle x_{u+1}, x_{u+1}\right\rangle+q^{2 k-n+u}[n-u-k]_{q}{ }^{2}\left\langle x_{u}, x_{u}\right\rangle}{\left\langle x_{u}, x_{u}\right\rangle+q^{2 k-n+u-1}[n-u-k+1]_{q}{ }^{2}\left\langle x_{u-1}, x_{u-1}\right\rangle} \\
& =\frac{\frac{\left\langle x_{u+1}, x_{u+1}\right\rangle}{\left\langle x_{u}, x_{u}\right\rangle}+q^{2 k-n+u}[n-u-k]_{q}{ }^{2}}{\left.1+q^{2 k-n+u-1}[n-u-k+1]_{q}{ }^{2} \frac{\left\langle x_{u-1}, x_{u-1}\right\rangle}{\left\langle x_{u}, x u\right.}\right\rangle} \\
& =\frac{q^{k}[u+1-k]_{q}[n-k-u]_{q}+q^{2 k-n+u}[n-u-k]_{q}{ }^{2}}{1+\frac{q^{2 k-n+u-1}[n-u-k+1]_{q}{ }^{2}}{q^{k}[u-k]_{q}[n-k-u+1]_{q}}} \\
& =q^{k+1}[u-k]_{q}[n-k-u]_{q}\left(\frac{[n-k-u]_{q}+q^{n-k-u}[u+1-k]_{q}}{[n-k-u+1]_{q}+q^{n-k-u+1}[u-k]_{q}}\right) \\
& =q^{k+1}[u-k]_{q}[n-k-u]_{q} .
\end{aligned}
$$


Since $\theta_{n}$ is an isomorphism, doing the procedure above for every SJC in $J_{q}(n)$ we get an orthogonal SJB of $V\left(B_{q}(n)\right) \oplus W(0)$ satisfying (3). That completes the proof.

Example We now work out a small example to illustrate the use of Theorem 1.3 to explicitly write down orthogonal SJB's. Let $q=2$. We consider orthogonal SJB's of $V\left(B_{2}(n)\right)$, for $n=0,1,2,3$. To save space we only write down the starting elements of each of the SJC's. We also drop the symbol $\mathcal{S}$ (for span) from the previous example.

(i) $n=0$ : There is only one SJC and its starting element is the zero subspace [].

(ii) $n=1$ : There is only one SJC and its starting element is the zero subspace [0].

(iii) $n=2$ : The unique SJC in item (ii) above will give rise to two SJC's in $V\left(B_{2}(2)\right)$ with starting elements (using (26) and (27))

$$
\left[\begin{array}{l}
0 \\
0
\end{array}\right] \text { and }-2\left[\begin{array}{l}
1 \\
0
\end{array}\right]+\left[\begin{array}{l}
0 \\
1
\end{array}\right]+\left[\begin{array}{l}
1 \\
1
\end{array}\right] .
$$

The unique SJC in item (i) above will give rise to a further SJC in $V\left(B_{2}(2)\right)$ with starting element (using the nontrivial character of $H(2,2)$ )

$$
\left[\begin{array}{l}
0 \\
1
\end{array}\right]-\left[\begin{array}{l}
1 \\
1
\end{array}\right]
$$

(iv) $n=3$ : Take the $\mathrm{SJC}$ in item (iii) above starting at $\left[\begin{array}{l}0 \\ 0\end{array}\right]$. This will give rise to two SJC's in $V\left(B_{2}(3)\right)$ starting at (using (26) and (27))

$$
\left[\begin{array}{l}
0 \\
0 \\
0
\end{array}\right] \text { and }-4\left\{\left[\begin{array}{l}
1 \\
0 \\
0
\end{array}\right]+\left[\begin{array}{l}
0 \\
1 \\
0
\end{array}\right]+\left[\begin{array}{l}
1 \\
1 \\
0
\end{array}\right]\right\}+3\left\{\left[\begin{array}{l}
0 \\
0 \\
1
\end{array}\right]+\left[\begin{array}{l}
1 \\
0 \\
1
\end{array}\right]+\left[\begin{array}{l}
0 \\
1 \\
1
\end{array}\right]+\left[\begin{array}{l}
1 \\
1 \\
1
\end{array}\right]\right\} \text {. }
$$

Now consider the SJC in item (iii) above starting at $-2\left[\begin{array}{l}1 \\ 0\end{array}\right]+\left[\begin{array}{l}0 \\ 1\end{array}\right]+\left[\begin{array}{l}1 \\ 1\end{array}\right]$. This will give rise to a further $\mathrm{SJC}$ in $V\left(B_{2}(3)\right)$ starting at (using (25))

$$
-2\left[\begin{array}{l}
1 \\
0 \\
0
\end{array}\right]+\left[\begin{array}{l}
0 \\
1 \\
0
\end{array}\right]+\left[\begin{array}{l}
1 \\
1 \\
0
\end{array}\right]
$$

Similarly, the SJC in item (iii) above starting at $\left[\begin{array}{l}0 \\ 1\end{array}\right]-\left[\begin{array}{l}1 \\ 1\end{array}\right]$ will give rise to yet another SJC in $V\left(B_{2}(3)\right)$ starting at (using (25))

$$
\left[\begin{array}{l}
0 \\
1 \\
0
\end{array}\right]-\left[\begin{array}{l}
1 \\
1 \\
0
\end{array}\right]
$$


Finally, the unique SJC in item (ii) above will give rise to three further SJC's in $V\left(B_{2}(3)\right)$ with starting elements (using the three nontrivial characters of $H(3,2)$ )

$$
\begin{aligned}
& {\left[\begin{array}{l}
0 \\
0 \\
1
\end{array}\right]-\left[\begin{array}{l}
1 \\
0 \\
1
\end{array}\right]+\left[\begin{array}{l}
0 \\
1 \\
1
\end{array}\right]-\left[\begin{array}{l}
1 \\
1 \\
1
\end{array}\right],} \\
& {\left[\begin{array}{l}
0 \\
0 \\
1
\end{array}\right]+\left[\begin{array}{l}
1 \\
0 \\
1
\end{array}\right]-\left[\begin{array}{l}
0 \\
1 \\
1
\end{array}\right]-\left[\begin{array}{l}
1 \\
1 \\
1
\end{array}\right],} \\
& {\left[\begin{array}{l}
0 \\
0 \\
1
\end{array}\right]-\left[\begin{array}{l}
1 \\
0 \\
1
\end{array}\right]-\left[\begin{array}{l}
0 \\
1 \\
1
\end{array}\right]+\left[\begin{array}{l}
1 \\
1 \\
1
\end{array}\right] .}
\end{aligned}
$$

We now consider the application of Theorem 1.3 to the Bose-Mesner algebra of the Grassmann scheme of $m$-dimensional subspaces. For convenience we assume $0 \leqslant m \leqslant n / 2$. We do not define this algebra here but instead work with the well known characterization that it equals the commutant of the $G L(n, q)$-action on $V\left(B_{q}(n)_{m}\right)$. For the proof of the following result see [15, Chapter 29] where the $q=1$ case is proven. The same proof works in general.

Theorem 3.1. Let $0 \leqslant m \leqslant n / 2$. Then $V\left(B_{q}(n)_{m}\right)$ is a multiplicity free $G L(n, q)$-module with $m+1$ distinct irreducible summands.

Thus End $\left.\operatorname{EL}_{G(n, q)}\left(V\left(B_{q}(n)_{m}\right)\right)\right)$ is a commutative $*$-algebra with dimension $m+1$ and so can be unitarily diagonalized.

Theorem 3.2. Let $0 \leqslant m \leqslant n / 2$. Define $J_{q}(n, m)=\left\{v \in J_{q}(n): r(v)=m\right\}$. Then $J_{q}(n, m)$ is a common orthogonal eigenbasis for the elements of $\left.\operatorname{End}_{G L(n, q)}\left(V\left(B_{q}(n)_{m}\right)\right)\right)$.

Proof For $i=0,1, \ldots, m$ and $k=0,1, \ldots, i$ define

$$
\begin{gathered}
J_{q}(n, i, k)=\left\{v \in J_{q}(n): r(v)=i \text { and the Jordan chain containing } v\right. \\
\text { starts at rank } k\} .
\end{gathered}
$$

Let $W_{q}(n, i, k)$ be the subspace spanned by $J_{q}(n, i, k)$. Then we have an orthogonal direct sum decomposition

$$
V\left(B_{q}(n)_{i}\right)=\oplus_{k=0}^{i} W_{q}(n, i, k) .
$$

Clearly $\operatorname{dim}\left(W_{q}(n, i, k)\right)=\left[\begin{array}{l}n \\ k\end{array}\right]_{q}-\left[\begin{array}{c}n \\ k-1\end{array}\right]_{q}$.

We shall now show that, for $i=0,1, \ldots, m, W_{q}(n, i, k), k=0,1, \ldots, i$ are $G L(n, q)$ submodules of $V\left(B_{q}(n)_{i}\right)$. We do this by induction on $i$, the case $i=0$ being clear.

Assume inductively that $W_{q}(n, i-1,0), \ldots, W_{q}(n, i-1, i-1)$ are $G L(n, q)$-submodules, where $i<m$. Since $U_{n}$ is $G L(n, q)$-linear, $U_{n}\left(W_{q}(n, i-1, j)\right)=W_{q}(n, i, j), 0 \leqslant j \leqslant i-1$ are $G L(n, q)$-submodules. Since

$$
W_{q}(n, i, i)=V\left(B_{q}(n)_{i}\right) \cap\left(\oplus_{k=0}^{i-1} W_{q}(n, i, k)\right)^{\perp}
$$


it follows that $W_{q}(n, i, i)$ is a $G L(n, q)$-submodule.

We now have from Theorem 3.1 that (28) is the decomposition of $V\left(B_{q}(n)_{i}\right)$ into distinct irreducible modules. The result follows.

We can also determine the eigenvalues of the elements of $\left.\operatorname{End}_{G L(n, q)}\left(V\left(B_{q}(n)_{m}\right)\right)\right)$ using (3). More generally, we can explicitly block diagonalize $\operatorname{End}_{G L(n, q)}\left(V\left(B_{q}(n)\right)\right)$. We refer to [27] for details.

Finally, we pose a bijective proof problem on the spanning trees of the Grassmann and Johnson graphs. Actually, this application only requires the existence of an orthogonal SJB satisfying (3) and not the actual construction from the present paper.

The number of spanning trees of a graph $G$ is called the complexity of $G$ and denoted $c(G)$. The number of rooted spanning trees (i.e., a spanning tree plus a choice of a vertex as a root) of $G$ is denoted $\bar{c}(G)$.

Let $0 \leqslant m \leqslant n / 2$. The Johnson graph $C(n, m)$ is defined to be the graph with $B(n)_{m}$, the set of all subsets in $B(n)$ of cardinality $m$, as the vertex set and with two vertices $X, Y \in B(n)_{m}$ connected by an edge iff $|X \cap Y|=m-1$.

Let $0 \leqslant m \leqslant n / 2$. The Grassmann graph $C_{q}(n, m)$ is defined to be the graph with vertex set $B_{q}(n)_{m}$, and with two vertices $X, Y \in B_{q}(n)_{m}$ connected by an edge iff $\operatorname{dim}(X \cap$ $Y)=m-1$.

Let $T_{q}(n, m)$ and $T(n, m)$ denote, respectively, the set of rooted spanning trees of $C_{q}(n, m)$ and $C(n, m)$.

For $X \in B_{q}(n)_{k}, X^{\prime} \in B_{q}(n)_{k-1}, 1 \leqslant k \leqslant n$ define

$$
\begin{aligned}
\mathcal{U D}(X) & =\left\{(Y, Z) \in B_{q}(n)_{k-1} \times B_{q}(n)_{k} \mid X \supseteq Y \subseteq Z\right\}, \\
\mathcal{D U}\left(X^{\prime}\right) & =\left\{\left(Y^{\prime}, Z^{\prime}\right) \in B_{q}(n)_{k} \times B_{q}(n)_{k-1} \mid X^{\prime} \subseteq Y^{\prime} \supseteq Z^{\prime}\right\} .
\end{aligned}
$$

Theorem 3.3. Let $0 \leqslant m \leqslant n / 2$. The sets

$$
T_{q}(n, m) \times \prod_{X \in B_{q}(n)_{m-1}} \mathcal{D} \mathcal{U}(X) \quad \text { and } \quad T_{q}(n, m-1) \times \prod_{X \in B_{q}(n)_{m}} \mathcal{U D}(X)
$$

have the same cardinality.

Proof We give an algebraic proof. For $X \in B_{q}(n)_{k}, X^{\prime} \in B_{q}(n)_{k-1}, 1 \leqslant k \leqslant n$ note that

$$
|\mathcal{U D}(X)|=\left|\mathcal{D U}\left(X^{\prime}\right)\right|=[k]_{q}[n-k+1]_{q} .
$$

Now, using the existence of an orthogonal SJB of $V\left(B_{q}(n)\right)$ satisfying (3) it was proved in [26] that the Laplacian eigenvalues of $C_{q}(n, m)$ are $[k]_{q}[n-k+1]_{q}, k=0,1, \ldots, m$ with respective multiplicities $\left[\begin{array}{l}n \\ k\end{array}\right]_{q}-\left[\begin{array}{c}n \\ k-1\end{array}\right]_{q}$. It now follows from the matrix-tree theorem (see [4]) that

$$
\bar{c}\left(C_{q}(n, m)\right)=\prod_{k=1}^{m}\left([k]_{q}[n-k+1]_{q}\right)^{\left[\begin{array}{l}
n \\
k
\end{array}\right]_{q}-\left[\begin{array}{c}
n \\
k-1
\end{array}\right]_{q}} .
$$


It follows that the sets in the statement of the theorem have the same cardinality.

The proof given above also works for the following result. We use similar notations as above.

Theorem 3.4. Let $0 \leqslant m \leqslant n / 2$. The sets

$$
T(n, m) \times \prod_{X \in B(n)_{m-1}} \mathcal{D} \mathcal{U}(X) \quad \text { and } \quad T(n, m-1) \times \prod_{X \in B(n)_{m}} \mathcal{U} \mathcal{D}(X)
$$

have the same cardinality.

For $m=1$, Theorem 3.4 gives $n|T(n, 1)|=n^{n}$, a result for which there is a celebrated bijective proof [16].

Problem Find bijective proofs of Theorems 3.3 and 3.4.

Recently, a related open problem, that of finding a combinatorial proof of the product formula for the complexity of the hypercube was solved in [3].

\section{References}

[1] C. Bachoc, F. Vallentin and A. Passuello, Bounds for projective codes from semidefinite programming, Advances in Math. of Communications, 7:127-145 (2013).

[2] F. Bergeron, Feedback by Francois Bergeron to the paper "binomial(5,2) proofs that binomial $(n, k) \leqslant \operatorname{binomial}(n, k+1)$ if $k<n / 2$, by D. Zeilberger, Personal journal of Shalosh B. Ekhad and Doron Zeilberger, available at http://www . math.rutgers . edu/ zeilberg/mamarim/mamarimhtml/trivialFB.html

[3] O. Bernardi, On the spanning trees of the hypercube and other products of graphs, Electronic J. Comb., 19(4):\#P51 (2012).

[4] A. E. Brouwer, and W. H. Haemers, Spectra of graphs, Springer, 2012.

[5] T. Ceccherini-Silberstein, F. Scarabotti, and F. Tolli, Harmonic analysis on finite groups, Cambridge University Press, 2008.

[6] P. Delsarte, Association schemes and t-designs in regular semilattices, J. Combinatorial Theory, Series A, 20:230-243 (1976).

[7] P. Delsarte, Hahn polynomials, discrete harmonics, and t-designs, SIAM J. Applied Math., 34:157-166 (1978).

[8] C. F. Dunkl, An addition theorem for some q-Hahn polynomials, Monatsh. Math., 85:5-37 (1978).

[9] K. Engel, Sperner theory, Cambridge University Press, 1997.

[10] J. Goldman and G. -C. Rota, The number of subspaces of a vector space, in Recent progress in Combinatorics (Proc. Third Waterloo Conf. on Combinatorics 1968), Academic Press:75-83 (1969).

[11] R. L Graham, S. Y. R. Li and W. W. Li, On the structure of t-designs, SIAM J. Alg. Discr. Methods, 1:8-14 (1980). 
[12] J. E. Graver and W. B. Jurkat, The module structure of integral designs, J. Comb. Theory, Ser. A, 15:75-90 (1973).

[13] J. R. Griggs, Sufficient conditions for a symmetric chain order, SIAM J. Applied Math., 32:807-809 (1977).

[14] S. Hitzemann and W. Hochstättler, On the combinatorics of Galois numbers, Discrete Math., 310:3551-3557 (2010).

[15] G. James, and M.Liebeck, Representations and Characters of Groups, Cambridge University Press, 2001.

[16] A. Joyal, Une théorie combinatoire des séries formelles, Advances in Math., 42:1-82 (1981).

[17] V. Kac and P. Cheung, Quantum Calculus, Springer-Verlag, 2002.

[18] D. E. Knuth, Combinatorial Matrices, in Selected papers on discrete mathematics, CSLI lecture Notes, 106, CSLI Publications, Stanford, CA:177-186 (2003).

[19] J. M. Marco and J. Parcet, On the natural representation of $S(\Omega)$ into $L^{2}(\mathcal{P}(\Omega))$ : discrete harmonics and Fourier transform, J. Comb. Theory, Ser. A, 100:153-175 (2002).

[20] J. M. Marco and J. Parcet, Laplacian operators and Radon transforms on Grassmann graphs, Monatsh. Math., 150:97-132 (2007).

[21] A. Nijenhuis, A. E. Solow and H. S. Wilf, Bijective methods in the theory of finite vector spaces, J. Comb. Theory, Ser. A, 37:80-84 (1984).

$[22]$ R. A. Proctor, Representations of $\mathfrak{s l}(2, \mathbb{C})$ on posets and the Sperner property, SIAM J. Alg. Discr. Methods, 3:275-280 (1982).

[23] A. Schrijver, New code upper bounds from the Terwilliger algebra and semidefinite programming, IEEE Tran. Information Theory, 51:2859-2866 (2005).

[24] N. Singhi, Tags on subsets, Discrete Math., 306:1610-1623 (2006).

[25] M. K. Srinivasan, Symmetric chains, Gelfand-Tsetlin chains, and the Terwilliger algebra of the binary Hamming scheme, J. Algebraic Comb., 34:301-322 (2011).

[26] M. K. Srinivasan, A positive combinatorial formula for the complexity of the q-analog of the n-cube, Electronic J. Comb., 19(2):\#P34 (2012).

[27] M. K. Srinivasan, Notes on explicit block diagonalization, in Combinatorial Matrix Theory and Generalized Inverses of Matrices, Springer:13-31 (2013).

[28] R. P. Stanley, Variations on differential posets, in Invariant Theory and Tableaux, volume 19 of IMA Vol. Math. Appl., Springer:145-165 (1990).

[29] R. P. Stanley, Enumerative Combinatorics - Volume 1, Second Edition, Cambridge University Press, 2012.

[30] P. Terwilliger, The incidence algebra of a uniform poset, in Coding theory and design theory, Part I, volume 20 of IMA Vol. Math. Appl., Springer:193-212 (1990).

[31] F. Vogt and B. Voigt, Symmetric chain decompositions of linear lattices, Comb., Prob. and Comp., 6:231-245 (1997). 